\title{
Hyperspectral Remote Sensing Data Recovery Via Adaptive Window Matching Method
}

\author{
http://dx.doi.org/10.3991/ijoe.v11i1.4235 \\ Yiliang Zeng ${ }^{1}$, Jinhui Lan ${ }^{1} *$, Chuanzhao Han $^{2}$, Libo Jiang ${ }^{1}$ and Xuefei Shi ${ }^{1}$ \\ ${ }^{1}$ University of Science and Technology Beijing, Beijing, P.R. China \\ ${ }^{2}$ Beijing Institute of Remote Sensing Information, Beijing, P.R. China
}

\begin{abstract}
HJ-1A satellite is often used to monitor environmental disaster and plays an important role in environmental changes. Because of the affection of various factors, certain band of HJ-1A hyperspectral remote sensing data is severe loss or distortion, which brings great difficulties for subsequent quantitative processing. A novel adaptive window matching algorithm, which can adjust intelligently size of matching window according to different local feature information of the image, is proposed for $\mathrm{HJ}-1 \mathrm{~A}$ satellite hyperspectral data recovery in this paper. The results show that the adaptive matching algorithm has a more superior performance than other algorithms in image quality index, column mean curve, and image correlation coefficient.
\end{abstract}

Index Terms-hyperspectral, data recovery, adaptive matching, wavelet analysis

\section{INTRODUCTION}

Hyperspectral remote sensing increased spectral dimension on basis of traditional two-dimensional remote sensing. Stripe noise is one of interference factors of hyperspectral remote sensing data, mainly caused by inconsistent responses of CCD detection units in hyperspectral imager [1]. It seriously affects on the data interpretation and information extraction. Horn et al. [2] firstly proposed histogram matching algorithm in 1979, which was applied to data recovery on Landsat MSS images. But the algorithm hypothesized that ground objects, which were detected by each sensor, must be equilibrium radiation distribution, and the algorithm could not adapt to complex scenes. Kautsky et al. [3] introduced a matching algorithm based on histogram smoothing, in which the histograms were modified by smooth processing. The matching precision was increased, but the algorithm failed to resolve complicated ground objects. Based on these theories, Wegener[4] put forward an improved histogram matching algorithm, and applied it to data recovery on multisensor remote sensing images. Gadallah et al .[5] proposed data recovery algorithm for multispectral images based on moment matching in 2000, then applied it into Landsat Thematic Mapper(TM) images. However, moment matching was under assumption that the output response of detector was linear, and the results were not perfect. Isostatic compensation method was proposed by Weinreb et al. [6] in 1989, which was used into data recovery for geostationary operational environmental satellite(GOES) images. This method [7] did not need histogram matching and moment matching, and used directly equalization curves of selected training site images to match other areas. But due to the artificial selection, the results had man-made errors. Crippen [8] introduced a new method based on spatial filtering to eliminate stripe noises on Landsat TM images by structuring special spatial filter, and achieved data recovery in spatial domain. Torres et al. [9] adopted wavelet transform to data recovery on multispectral images. Baker et al. [10] also proposed an improved wavelet analysis algorithm to deal with noise on multispectral images. but the algorithm had certain defects, such as data recovery was not complete, introduced new data loss, and so on. According to multispectral images, Lu Wei et al. [11] proposed cubic smooth spline to eliminate noise, which can settle the problem that other methods cannot filter noise adaptive and tested the algorithm with AVIRIS data, then results showed it had a good performance on data recovery for multispectral images. Stephan et al. [12] used principal component analysis (PCA) to de-noising, and completed data recovery finally. But it had a common effect, in addition, its calculation burden was too heavy. Hybrid Spmial-spectral wavelet shrinkage [13] was proposed to remove the noise in the derivative domain of hyperspectral data. Salah Bourennane et. Al [14] considered the spectral correlation problem and used the PARAFAC Model and Statistical Performance Analysis to eliminate noise. A Maximum a posteriori framework was established for hyperspectral images denoising by Shaolin Chen et. al [15].

In this paper, a recovery algorithm via adaptive window matching method is proposed to eliminate the stripe noise in HJ-1A hyperspectral remote sensing data. In order to better describe our algorithm, the rest of the paper is organized as follows. Section 2 gives the characteristics and causes of data loss or distortion in HJ-1A. The proposed algorithm flow, adaptability of the algorithm, and selection rule of threshold are described in Section 3. Section 4 evaluates our method by comparing with existing recovery methods.

\section{Characteristics And CAuses Of Data Loss Or DISTORTION}

\section{A. Characteristics of data loss or distortion}

For hyperspectral images of $\mathrm{HJ}-1 \mathrm{~A} / 1 \mathrm{~B}$ satellites, the main manifestation of data loss or distortion are fuzzy edge information of ground objects and stripe noises that are distributed along column direction. Stripe noises are distributed in the whole image and showed a periodic distribution approximately. Fig. 1 shows polluted image by stripe noises. Pixel values in contaminated areas are higher than adjacent areas, which can make it difficult to distinguish shape of the object. Furthermore, it will cause greater errors, even not to be normal proceeding, so it's significant to remove stripe noises. While fuzzy edge will 


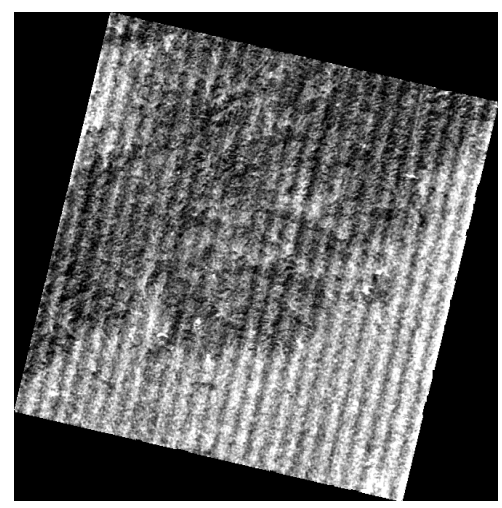

Figure 1. Data loss or distortion of hyperspectral images

decrease the accuracy of the image, which will make features not obvious and bring in great difficulties for subsequent quantitative processing as well.

\section{B. Causes of stripe noise}

Stripe noise is mainly produced by inconsistent responses of CCD detection units in hyperspectral imager. In addition, stripe noise is also caused by other reasons, such as some minor errors are caused by calibration, change of sensor response, mechanical movement and change of temperature of sensors. As for hyperspectral data of HJ-1A satellites, some bands are polluted so seriously that they must be recovered. By concrete analysis, reasons of response difference are as follows: 1) Inherent inhomogeneity of every CCD; 2) Influenced by CCD dark current; 3) Inconsistency by working state; 4) Influenced by external factors, and 5) Correction of CCD response function is incomplete and inaccuracy.

And the causes of making edge and texture of the ground object fuzzy include: 1) Influenced by working environment; 2) Minor errors caused by system calibration; 3) Temperature drift and 4) Errors in image coding, transmission.

\section{HyPERSPECTRAL DATA RECOVERY ALgORITHM}

\section{A. Algorithm flow}

In order to cope with the problem of poor local data recovery, this paper proposes a new matching algorithm of adaptive window, The core idea of the algorithm is that it can adjust intelligently size of matching window according to different local information to match two images. The flow diagram of adaptive matching algorithm is shown in Fig.2.

The first step is that calculating difference image of normal image and distortion image. The difference image can be obtained from $\nabla I=I_{c k}-I_{s z}$.

Where $I_{c k}$ and $I_{s z}$ are reference image and distortion image. The method can not only reduce computational work and simplify algorithm, but also ensure precision of the algorithm. Difference image is utilized to describe the local characteristic difference between reference image and distortion image. The $6 \times 6$ sliding window is selected and the variance of difference image is calculated in the window. Then variance is used to determine which kind of window (big window or small window) to match the images. Selection criteria will be presented in the next step.

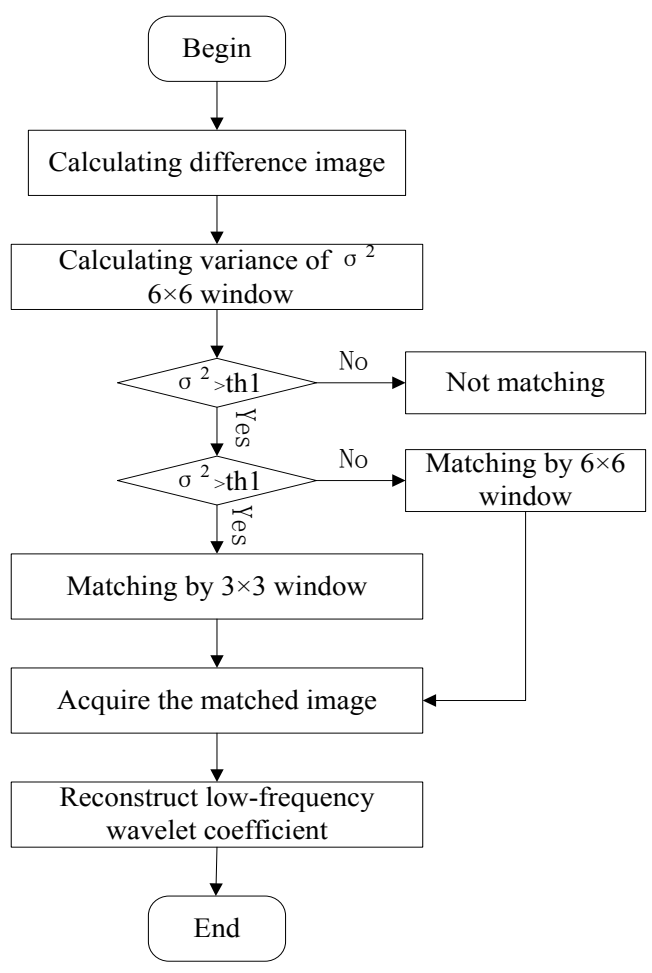

Figure 2. Flow diagram of adaptive iterative algorithm

The next step is that calculating pixel variance $\sigma^{2}$ in $6 \times 6$ sliding window, and compares with global threshold $t h 1$, if $\sigma^{2} \leq t h 1$, it means that there is tiny distinction between reference image and distortion image in the window, and it need not matching. But if $\sigma^{2}>t h 1$, it indicates two images have large difference, so it needs to be matched.

Then $\sigma^{2}$ is compared with local threshold th2, if $\sigma^{2} \leq t h 2$, which shows that deviation is smaller, matching by $6 \times 6$ window will meet the requirement. But if $\sigma^{2}>$ th 2 , which indicates deviation is larger, $6 \times 6$ window is unable to meet the requirement, we must divide big window further, a big $6 \times 6$ window will be divided into 4 small $3 \times 3$ windows, then matching two images in 4 small $3 \times 3$ windows.

After adaptive matching algorithm, the matched image has some defects, which mainly shown that certain homogeneous ground object have data missing. Therefore, lowfrequency wavelet coefficient is reconstruct to settle data missing in our algorithm.

The biggest advantage of the algorithm has adaptability, it can adjust the size of matching window according to different local information.

\section{B. Adaptability of the algorithm}

In the process of putting forward the algorithm, the first problem is to select parameter to measure local information. In general, optional parameters include mean and variance. In our algorithm, the variance parameter is chosen. The reason is that mean belongs to first moment, which only reflects the local average gray. So information is limited. If adopting it to measure local feature, we can't obtain better restoration results. But variance belongs to second-order moment, which is stronger than first moment in describing local information. So we decide to utilize variance parameter in the algorithm. 
Calculating the difference of local variance between two images, Variances of two images can be calculated respectively in the same window, then subtracting; another method is that calculating difference image of two images, and then requiring local variance of the difference image. The latter is utilized in adaptive matching algorithm. In which the difference image is $\nabla I$, it can be expressed as follows: $\nabla I=I_{c k}-I_{s z}, I_{c k}$ and $I_{s z}$ are reference image and distortion image. The method can not only reduce computational work and simplify algorithm, but also ensure precision of the algorithm. 9th-band image (distortion image) and 108th-band image (reference image) of hyperspectral data at a certain area and their difference image are given in Fig.3.

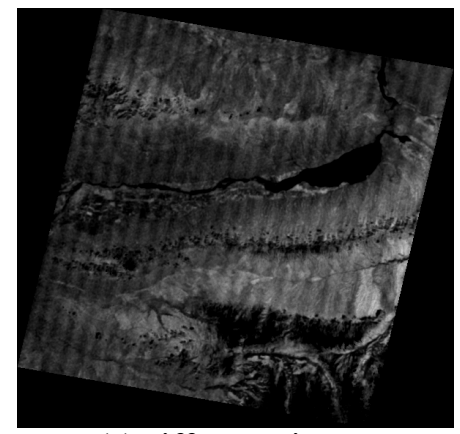

(a)Difference image

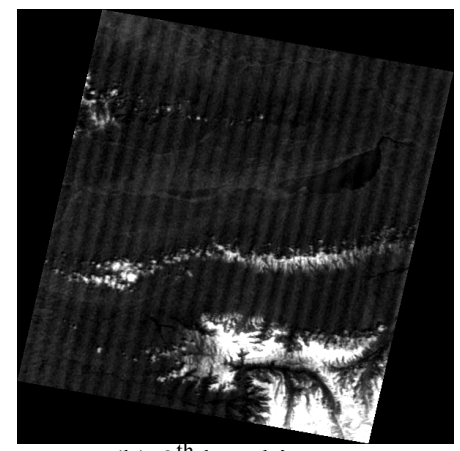

(b) $9^{\text {th }}$ band image

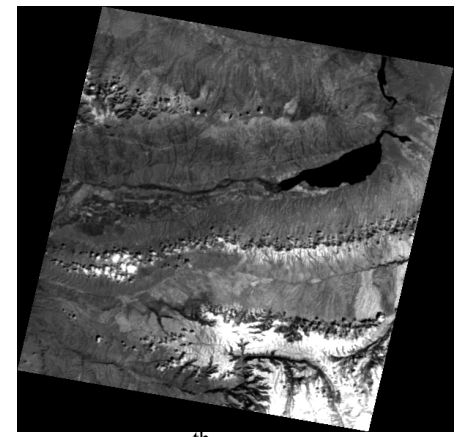

(c) $108^{\text {th }}$ band image

Figure 3. Difference image of distortion image and reference image

The next step is selecting different size of windows by variance to match the images. Traditional methods only use one window to match images, these methods are feasible in the case of low require. However, traditional methods can't reach satisfactory effect for data recovery. If applying small $3 \times 3$ window simply, new data loss is introduced. Besides, some regions in the image will become fuzzy, even it's not obvious. But if adopting big $6 \times 6$ window alone, the overall effect is extremely terrible. First, stripe noises are not removed wholly. Second, fuzzy edge of ground object has not been restored completely, and it can be said that the result is worse. However, the big window has an advantage that it has a perfect performance on data recovery of large-scale homogeneous ground objects, gets a nice effect, and there is not new data loss in the bottom left of the image.

For combining advantages of big window with advantage and small window, the algorithm with certain adaptive ability is needed, which can adjust the size of matching window according to different local information of the image. In this paper, global threshold and local threshold are set to realize the adaptive ability of the proposed algorithm.

\section{Selection rule of threshold}

The threshold selection process, which has been described in Section 2.A, can be expressed clearly by the following coordinate axis in Fig.4.

0 th1 the

Figure 4. Threshold region representation

If $\sigma^{2} \in(0, t h 1]$, not matching the images. If $\sigma^{2} \in(t h 1, t h 2]$,matching the images by big $6 \times 6$ window. And if $\sigma^{2} \in(t h 2,+\infty]$, matching by small $3 \times 3$ window.

It is necessary to understand approximately function of threshold before determining threshold selection formula. In matching algorithm of adaptive window, the role of threshold is selecting a matching area needed. Black border around the image must be out of matching range, as the same time, some local homogeneous area needn't match. So, the value of $t h 1$ cannot be too large, otherwise, it's easy to miss some area that need to be matched; the value of $t h 1$ is too small, which will increase the computational complexity but couldn't make the result better. Combining above rules with the actual situation, the global threshold $t h 1$ can be represented as

$$
t h 1=\sqrt{\frac{2}{n} \cdot \sum_{i=1}^{n} \sigma_{i}^{2}}=\sqrt{\frac{2}{n} \cdot\left(\sigma_{1}^{2}+\sigma_{2}^{2}+\sigma_{3}^{2}+\ldots+\sigma_{n}^{2}\right)}
$$

In which $\sigma_{i}^{2}$ is the variance of the ith pixel value in $6 \times 6$ window, $\mathrm{n}$ represents number of $6 \times 6$ windows. The local threshold thl is the same as $t h 1$, it is defined as following form:

$$
\operatorname{th} 2=\sqrt{\frac{2}{n} \cdot \sum_{i=1}^{n} \theta_{i}^{2}}=\sqrt{\frac{2}{n} \cdot\left(\theta_{1}^{2}+\theta_{2}^{2}+\theta_{3}^{2}+\ldots+\theta_{n}^{2}\right)}
$$

where $\theta_{i}^{2}$ is the variance of the ith pixel value in $3 \times 3$ windows, $n$ represents number of $3 \times 3$ windows.

The reason why select the values of global threshold and local threshold is that we consider the effect of the two thresholds. If selecting the mean of variances as threshold, it will lose pertinence of the threshold. But the standard deviation is selected as the threshold, which also weak pertinence of the threshold. So, the aforesaid threshold formulas are required by standard deviation is multiplied by $\sqrt{2}$. The experimental results show that the thresholds have strong pertinence.

Choosing matching method is based on moment matching (the principle in the appendix) method, the first mo- 
ment (mean) and the second-order moment (variance) are used to match images. Different thresholds will acquire various sizes of window, the matching formula can be defined as follows:

$$
D N_{\text {adjusted }}=\left(D N_{\text {initial }}-\mu_{\text {sensor }}^{i}\right) \frac{\sigma_{\text {reference }}}{\sigma_{\text {sensor }}^{i}}+\mu_{\text {reference }}
$$

where $D N_{\text {initial }}$ and $D N_{\text {adjusted }}$ are separately standing for the initial pixel value and the matched pixel value. $\mu_{\text {initial }}$ represents the mean of pixel values in adaptive window; $\mu_{\text {reference }}$ is the mean of pixel values in reference image; $\sigma_{\text {initial }}$ denotes the standard deviation of pixel values in adaptive window; and $\sigma_{\text {reference }}$ corresponds to the standard deviation of pixel values in reference image.

\section{4. EXPERIMENTAL RESULTS AND ANALYSIS}

\section{A. Evaluation index}

Nowadays, there are two main ways to evaluate data recovery effect: subjective evaluation and objective evaluation. Subjective evaluation uses visual contrast to evaluate restoration effects. The advantage is visual and simple, but it can only qualitative evaluation and not quantitative evaluation. While objective evaluation achieves quantitative evaluation of the data recovery effect by calculating some parameter indexes.

There is a dedicated index of evaluation de-noising effect in the field of hyperspectral remote sensing image processing. That is image quality factor $I Q$, it can be obtained from

$$
\begin{aligned}
I Q=10 \log _{10}\left[\sum_{i=1}^{M} d_{R}^{2}[i] / \sum_{i=1}^{M} d_{E}^{2}[i]\right] \\
d_{R}[i]=m_{I R}[i]-m_{I}[i] \\
d_{E}[i]=m_{I E}[i]-m_{I}[i]
\end{aligned}
$$

in which $m_{I R}[i], m_{I E}[i]$ and $m_{I}[i]$ are the mean of every line in original stripe noise image, de- noising image and real surface image. If the value of $I Q$ is bigger, which indicates that de-noising effect is better; the value of $I Q$ is smaller, which shows that de-noising effect is worse; but if the value of $I Q$ is a negative, it means that the change of image is larger, the de-noising image has severe distortion.

This paper adopts two indexes to evaluate data recovery effect. One is correlation coefficient between recovered image and reference image, the other is column mean curve of recovered image. The correlation coefficient $H$ can be required by

$$
H=\frac{\sum_{i=1}^{M} \sum_{j=1}^{N}\left(X_{i j}-\mu_{X}\right)\left(Y_{i j}-\mu_{Y}\right)}{\sqrt{\left[\sum_{i=1}^{M} \sum_{j=1}^{N}\left(X_{i j}-\mu_{X}\right)^{2}\right]\left[\sum_{i=1}^{M} \sum_{j=1}^{N}\left(Y_{i j}-\mu_{Y}\right)^{2}\right]}}
$$

The size of correlation coefficient can evaluate data recovery effect, the bigger it is, the better restoration effect the algorithm will make, and the closer between recovered image and reference image. Otherwise, it indicates the recovery effect is worse.

In addition, the column mean curve also can evaluate data recovery effect. Data recovery effect is better when the column mean curve of recovered image matches the column mean curve of reference image closer. Otherwise, data recovery effect will become worse. The column mean should satisfy,

$$
m_{J}=\frac{1}{N} \sum_{i=1}^{M} X_{i J}
$$

\section{B. Experimental results}

In order to verify fully the validity of the algorithm in this paper, three hyperspectral images of $\mathrm{HJ}-1 \mathrm{~A} / 1 \mathrm{~B}$ satellite are selected to carry out three groups' experimentation. Three hyperspectral images have respectively mild, moderate and severe data distortion, Data recovery on the three hyperspectral images to test the abilities of removing stripe noises, recovering fuzzy edge of ground objects, regaining overall gray distribution of image.

\section{1) Data recovery of mild distortion image}

In the first experiment, the distortion image is disturbed mainly by stripe noises, and degree of vagueness is slighter at the edge of ground object. Besides, overall gray distribution of image is close to normal image, and the image is showed in Fig.5 (a). So the aim of choosing such image is that testing the ability of removing stripe noises.

In Fig.5, (a) represents original distortion image, (b) represents reference image, (c) is the image that utilize wavelet analysis method to remove stripe noises, (d) is the result of wavelet analysis method with histogram matching, this algorithm is called as "algorithm 1", (e) is acquired by wavelet domain recovery algorithm based on PDE variational model, this algorithm is called as "algorithm 2", and (f) is the result of adopting adaptive window matching method, this proposed algorithm is called as "algorithm 3".Calculating column mean curves of the images after getting de-noising images. Next, a series of parameter indexes is calculated to compare the performance of the three algorithms.

The difference of distortion image and reference image is that distortion image is contaminated severely by stripe noises, but edge information of ground objects have not obvious loss or distortion. So the first experiment uses for testing the ability of removing stripe noises. Fig.5 (d), (e) and (f) show three algorithms all can remove stripe noises effectively. The result of Fig.5 (c) shows that wavelet analysis method can eliminate stripe noises, but it can't recover contaminated information completely. It can be seen in the top left corner of Fig.5 (c), the edge of mountains have become considerable fuzzy. It shows that traditional algorithms can't recover hyperspectral data of the HJ-1A satellite. From Fig.5 (d), (e) and (f), the fuzzy edge of ground object in the top left corner has gotten a better recovery. But it is difficult to distinguish virtues or defect degree of three algorithms.

Therefore, some other parameters are taken to analysis quantitatively performance of each algorithm. The column means of reference image and the images that are disposed by three algorithms are calculated, the curves are shown as following in Fig.6. 


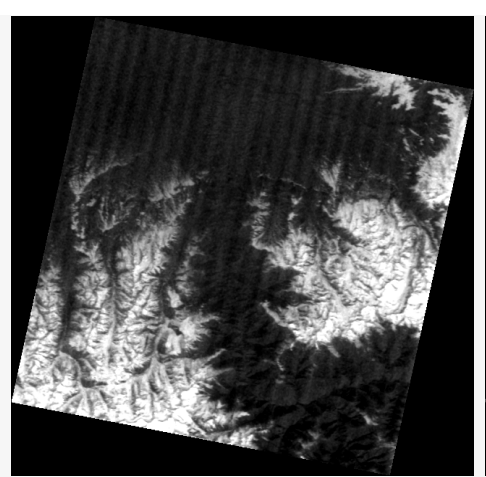

(a) Distortion image

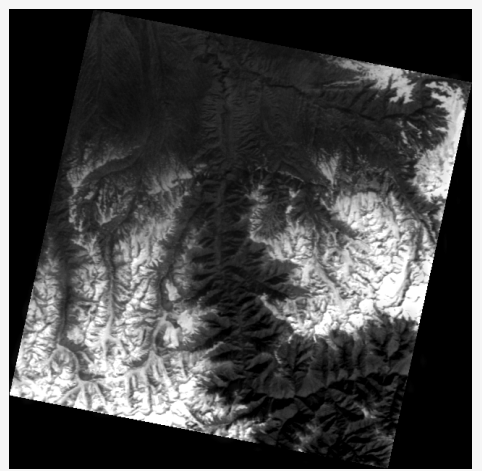

(d) Algorithm 1

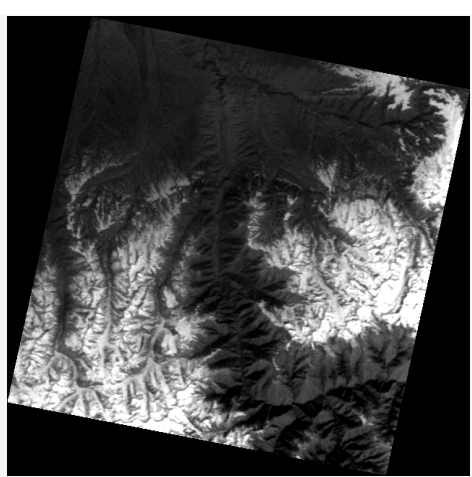

(b) Reference image

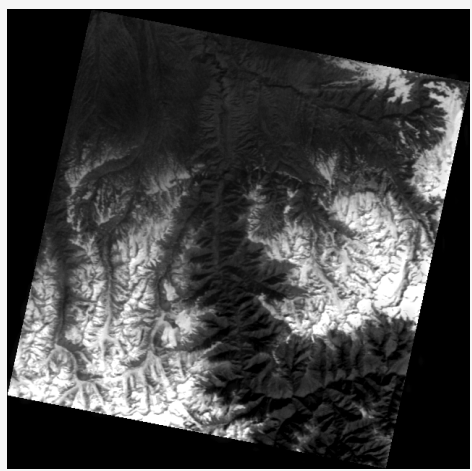

(e) Algorithm 2

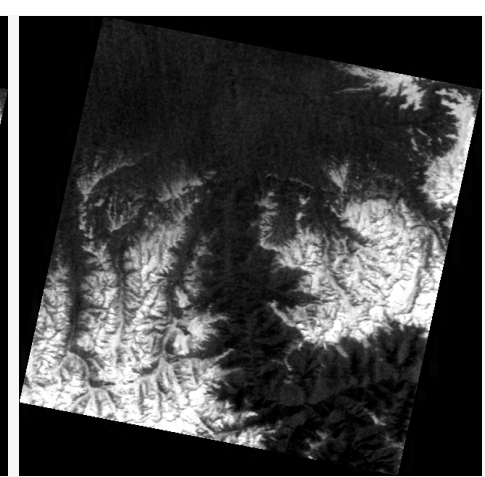

(c) De-noising image

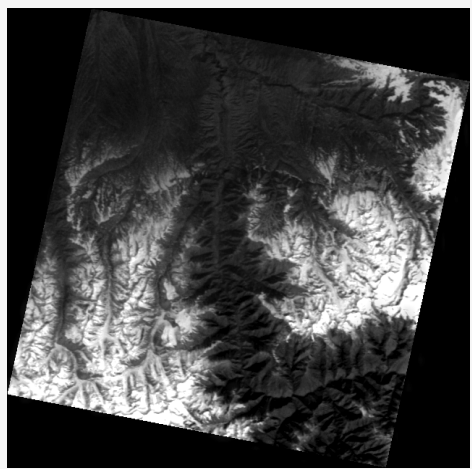

(f) Algorithm 3

Figure 5. Experiment results of mild distortion image

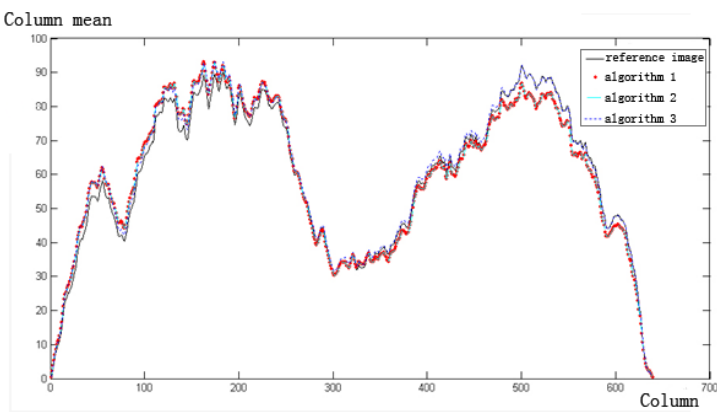

Figure 6. Column mean curve of the first experiment

In Fig.6, the algorithm 3 has the best restoration effect, most of regions are the almost same as the reference image except a few columns. As for algorithm 1 and algorithm 2, column mean is higher than normal value in the left half of the image, and it is lower in the another half of the image. Besides, the deviation in the left half of the image is bigger than another. At the same time, the effect of algorithm 1 and algorithm 2 has no difference.

It is not enough to offer a parameter index to evaluate the performance of three algorithms, and it is more convincing to adopt other parameter indexes. The results of other parameter indexes are given in Table I.

Various data of Table I shows that three algorithms have little difference on traditional evaluation parameters including $\mu$ and $\sigma$. Algorithm 3 has better performance on image quality factor $I Q$ than algorithm 1 and algorithm 2, and algorithm 1 and algorithm 2 have little difference. Three algorithms are better performance on peak signal to noise ratio (PSNR) than traditional algorithms, and PSNR of algorithm 3 is highest, while algorithm 1 and algorithm
TABLE I.

RESULTS OF OTHER PARAMETER INDEXES

\begin{tabular}{|l|c|c|c|c|c|}
\hline & $\boldsymbol{\mu}$ & $\boldsymbol{\sigma}$ & $\mathbf{I Q}$ & $\mathbf{P S N R} / \mathbf{d b}$ & $\mathbf{H}$ \\
\hline Reference Image & 59.673 & 171.409 & & & \\
\hline Distortion Image & 57.704 & 177.207 & & 26.804 & 0.988 \\
\hline De-noising Image & 57.552 & 176.045 & 0.578 & 27.382 & 0.989 \\
\hline Algorithm 1 & 59.642 & 171.424 & 4.517 & 31.321 & 0.995 \\
\hline Algorithm 2 & 59.639 & 171.428 & 4.520 & 31.324 & 0.995 \\
\hline Algorithm 3 & 61.115 & 174.174 & 9.689 & 36.494 & 0.999 \\
\hline
\end{tabular}

2 are close. Algorithm 3 is also superior to algorithm 1 and algorithm 2 in the correlation coefficient $H$, and algorithm 1 is the almost same as algorithm 2 .

By comparing column mean curve and different parameters, we can obtain the following conclusions: three algorithms all can complete the aim of data recovery on mild loss or distortion images, and get better de-noising effect. But the performance of adaptive window matching method is best, especially in column mean curve, image quality factor $I Q$, peak signal to noise ratio PSNR and image correlation coefficient $H$, and it is far superior to wavelet analysis method with histogram matching and wavelet domain recovery algorithm based on PDE variational model. The latter two algorithms have little difference on various evaluation parameters.

\section{2) Data recovery of severe distortion image}

In the second experiment, the selected image has some characteristics, including severe stripe noises, serious fuzzy edge of ground objects and having a big gray difference between distortion image and normal image. So the aim of the second experiment is that testing comprehen- 


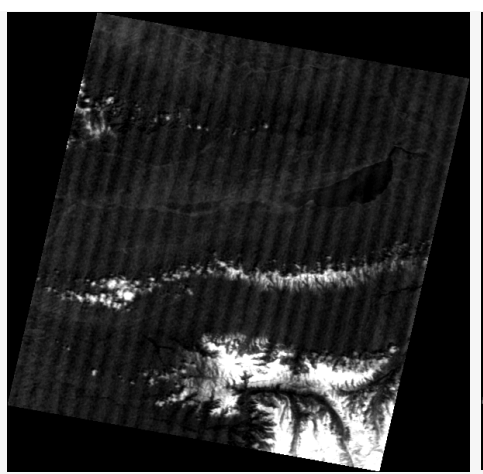

(a) Original image

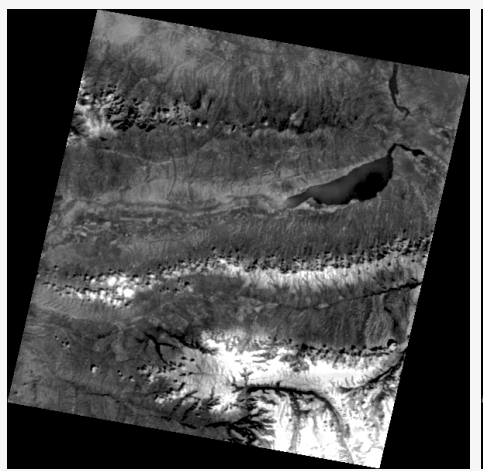

(d) Algorithm 1

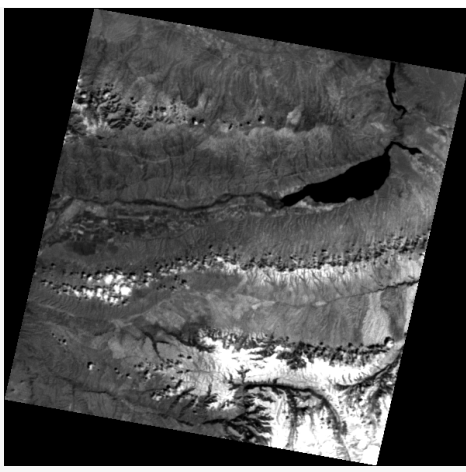

(b) Reference image

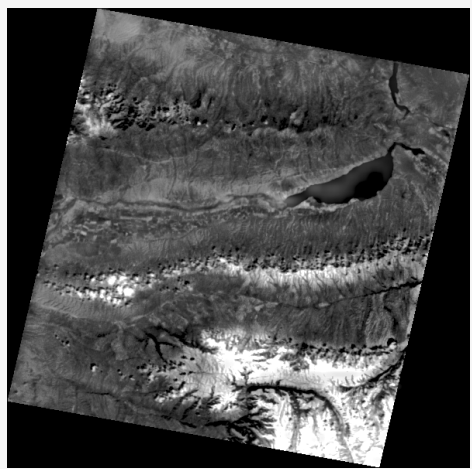

(e) Algorithm 2

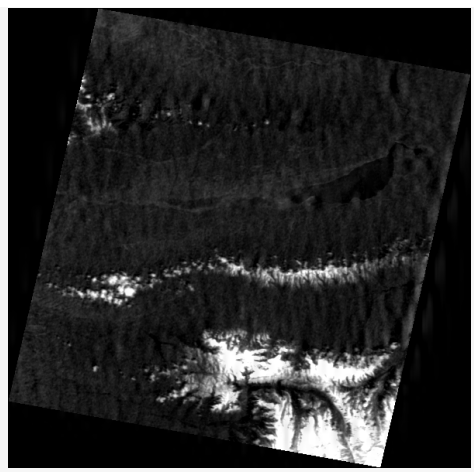

(c) De-noising image

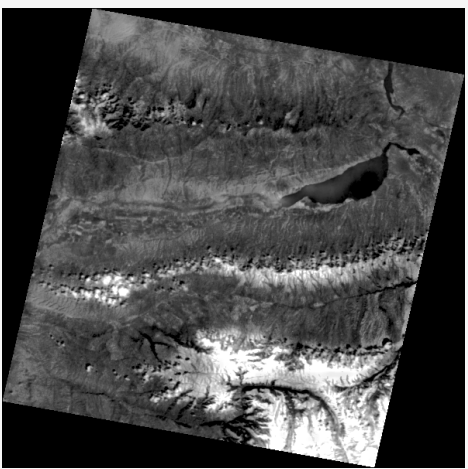

(f) Algorithm 3

Figure 7. Experiment results of severe distortion image

sive properties of the algorithms, such as recovering fuzzy edge of ground objects on the basis of removing stripe noises, adjusting gray distribution of the whole image etc.

Like the first experiment, in Fig.7, (a) represents original distortion image;(b) represents reference image;(c) is the image that utilize wavelet analysis method to remove stripe noises;(d) is "algorithm 1";(e) is "algorithm 2";(f) is "algorithm 3". The column mean curves of each image are calculated after getting de-noising images. And then, a series of parameter indexes is calculated to compare the performance of the three algorithms.

The distortion image has severe data loss and distortion, which mainly show in three aspects: (1)polluted severely by stripe noises;2(2an't identify ground objects by visual because of fuzzy edge;(3have a large gray distribution difference with the reference image. So the second experiment tests comprehensive properties of the algorithms by the results of solving aforesaid questions.

Experimental results show that traditional methods can barely remove stripe noises, even it will cause reforming phenomena, and it also can't recover fuzzy ground object edge, let alone adjust the gray distribution of the whole image. But in this paper, three algorithms can complete the task of data recovery. Not only can they eliminate stripe noises, but also recover edge information, even adjust gray distribution. The curves are shown in Fig.8.

In Fig.8, for algorithm 1 and algorithm 2, column mean is higher than normal value in the left half of the image, and it is lower in the right half of the image, but it has a better performance in the middle. Although the algorithm 3 has a little deviation in the middle, it is better than the two former algorithms. All in all, the algorithm 3 is best, and the effect of algorithm 1 and algorithm 2 has no difference.

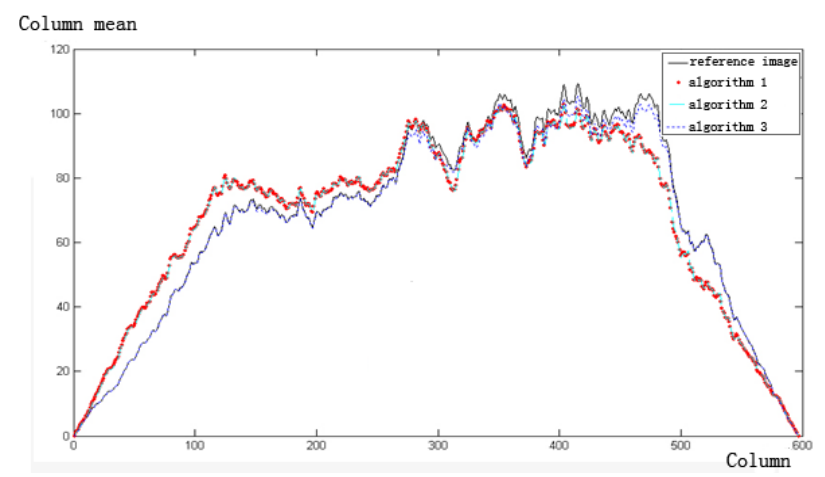

Figure 8. Column mean curve of the second experiment

TABLE II.

RESULTS OF OTHER PARAMETER INDEXES

\begin{tabular}{|l|c|c|c|c|c|}
\hline & $\boldsymbol{\mu}$ & $\boldsymbol{\sigma}$ & IQ & PSNR/db & H \\
\hline Reference Image & 67.156 & 141.573 & & & \\
\hline Distortion Image & 31.692 & 115.368 & & 14.119 & 0.812 \\
\hline De-noising Image & 57.552 & 114.034 & 0.104 & 14.223 & 0.819 \\
\hline Algorithm 1 & 67.154 & 141.570 & 5.999 & 20.118 & 0.914 \\
\hline Algorithm 2 & 67.129 & 141.592 & 6.142 & 20.260 & 0.917 \\
\hline Algorithm 3 & 65.945 & 135.008 & 17.179 & 31.298 & 0.995 \\
\hline
\end{tabular}

The results of other parameter indexes are given in Table II. It shows that three algorithms have little difference on traditional evaluation parameters including $\mu$ and $\sigma$; Algorithm 3 has better performance on image quality factor $I Q$ than the others, and algorithm 1 and algorithm 2 have little difference; three algorithms are better performance on peak signal to noise ratio (PSNR) than traditional algorithms, and PSNR of algorithm 3 is highest, the 
others are close; algorithm 3 is also superior to algorithm 1 and algorithm 2 in the correlation coefficient $H$, and algorithm 1 is the almost same as algorithm 2 .

So we can conclude the following conclusions: the three algorithms all can complete the aim of data recovery on severe loss or distortion images, get better de-noising effect and edge restoration effect. But the performance of the adaptive window matching method is best, especially in column mean curve, image quality factor $I Q$, peak signal to noise ratio PSNR and image correlation coefficient $H$, and it is far superior to wavelet domain recovery algorithmsogram matchingvocationalt domain recovery algorithm based on PDE variational model. The latter two algorithms have little difference on various evaluation parameters.

\section{CONCLUSION}

Traditional algorithms only recover the images that are contaminated by stripes noises, but they will not receive better effect to recover hyperspectral data of HJ-1A satellite. The reason is that how to recover fuzzy edge and texture information isn't considered. A matching algorithm of adaptive window is proposed in this paper, which can adjust intelligently size of matching window according to different local feature information of the image, besides, recover the data that are polluted by stripe noises and edge and texture information as much as possible. The effectiveness of the algorithm is verified by the abilities of removing stripe noises, recovering fuzzy edge and overall gray distribution of the image. The matching algorithm of adaptive window is superior to wavelet analysis method with histogram matching and wavelet domain recovery algorithm based on PDE variational model in objective and quantitative parameter indexes, Not only does it success in accomplishing data recovery, but also it enhances edge and texture information, and provides data support to subsequent quantitative processing on hyperspectral data.

\section{REFERENCES}

[1] Adam C, Zelinski, Vivek K G. "Denoising Hyperspectral Imagery and Recovering Junk Bands using Wavelets and Sparse Approximation,". IEEE Intemational Geoscience and Remote Sensing Symposium, Vol.104, pp. 387-390, 2006.

[2] Horn B K P, Woodham R J. "Destriping Landsat MSS Imagery by Histogram Modification," Computer Graphics and Image Processing, Vol.10, pp. 69-83,1979. http://dx.doi.org/10.1016/0146664X(79)90035-2

[3] Kautsky J, Nichols N K, Jupp D L B. "Smoothed Histogram Modification for Image Processing," Computer Vision Graphics and Image Processing, Vol.26, pp. 271-291, 1984. http://dx.doi.org/10.1016/0734-189X(84)90213-5

[4] Wegener M, Destriping "Multiple Sensor Imagery by Improved Histogram Matching," International Journal of Remote Sensing, Vol.11, No.5, pp. 859-875, 1990. http://dx.doi.org/10.1080/ 01431169008955060

[5] Gadallah F L, Csillag F. "Destriping Multisensor Imagery with Moment Matching,". International Journal of Remote Sensing, Vol.21, No.12, pp.2505-2511, 2000. http://dx.doi.org/10.1080/ 01431160050030592

[6] Weinreb M P, Xie R, "Lienesch J H. Destriping GOES Images by Matching Empirical Distribution Fuctions," Remote Sensing of Environment, Vol.29, No.2, pp. 185-195, 1989. http://dx.doi.org/10.1016/0034-4257(89)90026-6

[7] Giovani Corsini, Marco Diani, Thomas Walzel. "Striping Removal in MOS-B Data,". IEEE Transactions on Geoscience and Remote Sensing, Vol.38, No.3, pp. 1439-1446, 2000. http://dx.doi.org/10.1109/36.843038

[8] R. E. Crippen. "A simple spatial filtering routine for the cosmetic removal of scan-line noise from Landsat TM P-tape imagery,"
Photogramm. Eng.Remote Sens.,Vol. 55, No.3, pp. 327-331, 1989.

[9] J. Torres and S. O. Infante, "Wavelet analysis for the elimination of striping noise in satellite images," Opt. Eng, Vol.40, No.7, pp.1309-1314, 2001. http://dx.doi.org/10.1117/1.1383996

[10] Backer S D, Piurica A, and Huysmans B. "Denoising of multicomponent images using wavelet least-squares estimators," Image and Vision Computing, Vol.26, No.7, pp. 1038-1051, 2008. http://dx.doi.org/10.1016/j.imavis.2007.11.003

[11] Wei Lu, Xuchu Yu, and Juan Liu. "The Application of Cubic Smooth Spline in Noise Filtering to Hyperspectral Data," Journal of Institute of Surveying and Mapping,Vol. 22, No.1, pp. 11-13, 2005.

[12] Stephan K, Hibbitts C A, and Hoffmann H. "Reduction of instrument-dependent noise in hyperspectral image data using the principal component analysis: Applications to Galileo NIMS data," Planetary and Space Science, Vol.56, No.3, pp. 406-419, 2008. http://dx.doi.org/10.1016/j.pss.2007.11.021

[13] Othman H, Qian S E. "Noise reduction of hyperspectral imagery using hybrid Spmial-spectral derivative domain wavelet shrinkage," IEEE Transactions on Geoscience and Remote Sensing, Vol.44, No.2, pp. 397 408, 2006. http://dx.doi.org/10.1109/TGRS. $\underline{2005.860982}$

[14] Xuefeng Liu, Bourennane, S. Fossati, C. "Denoising of Hyperspectral Images Using the PARAFAC Model and Statistical Performance Analysis," IEEE Transactions on Geoscience and Remote Sensing, Vol.50, No.10, pp. 3717 - 3724, 2012. http://dx.doi.org/10.1109/TGRS.2012.2187063

[15] ShaoLin Chen , Xi-Yuan Hu and Si-Long Peng . "Hyperspectral Imagery Denoising Using a Spatial-Spectral Domain Mixing Prior," Journal of computer science and technology, Vol.27, No.4, pp. 851-861, 2012. http://dx.doi.org/10.1007/s11390-012-1269-1

\section{AUTHORS}

Yiliang Zeng received the B.E. degree from University of Science and Technology Beijing (USTB), Beijing, China in 2009. Currently, he is pursuing a $\mathrm{PhD}$ degree in control science and engineering at the University of Science and Technology Beijing, Department of Instrument Science and Technology, School of Automation and Electrical Engineering, Beijing 100083, China. (e-mail: ustbzengyiliang@163.com).

Jinhui Lan. obtained the PhD degree from Beijing Institute of Technology, China in 1998. From August 2002 to February 2004, she was a Visiting Academic at Deakin University, Australia. Since July 2008, she has been a Professor at the University of Science and Technology Beijing, Department of Instrument Science and Technology, School of Automation and Electrical Engineering, Bejing 100083, China. From February 2013 to August 2013, she was a visiting scholar at University of Wisconsin-Madison, USA. (e-mail: ustblanjinhui@gmail.com).

Libo Jiang is is pursuing a master's degree in control science and engineering at the University of Science and Technology Beijing, Department of Instrument Science and Technology, School of Automation and Electrical Engineering, Beijing 100083, China. (e-mail: 236009611@qq.com).

Chuanzhao Han is with Beijing Institute of Remote Sensing Information, Beijing 100192, China.

Xuefei Shi is with Department of Instrument Science and Technology, School of Automation and Electrical Engineering, University of Science and Technology Beijing, Beijing 100083, P.R. China.

This work was supported by the National Natural Science Foundation of China (Grant No. 61174181) and National High Technology Research and Development Program of China (863 Program, Grant No. 2012AA12A302-4). Submitted 30 October 2014. Published as resubmitted by the authors 25 January 2015. 